\title{
ASYMPTOTIC PROPERTY OF SOLUTIONS ON NONAUTONOMOUS LOTKA-VOLTERRA MODEL FOR $N$-COMPETING SPECIES
}

\author{
KUNIHIKO TANIGUCHI
}

Abstract. We consider nonautonomous $N$-dimensional generalized Lotka-Volterra competition systems. Under certain conditions we show that the set of values of solutions starting from any compact set tends to a set of measure zero. Our results give generalizations of previous ones.

Mathematics subject classification (2010): 34D05, 92D25.

Keywords and phrases: Lotka-Volterra systems, Nonautonomous system, competition system, permanence.

\section{REFERENCES}

[1] S. Ahmad, A. C. LAZER, On a property of nonautonomous Lotka-Volterra competition model, Nonlinear Anal., 37 (1999), 603-611.

[2] S. Ahmad, A. C. LAZER, On the nonautonomous N-competing species problem, Appl. Anal., 57 (1995), 309-323.

[3] P. Billingsley, Probability and measure, John Wiley \& Sons, New York, 1979.

[4] W. A. Coppel, Stability and Asymptotic Behavior of Differential Equations, P. C. Heath and Company, Boston, 1965.

[5] J. Hofbauer, K. Sigmund, Evolutionary Games and Population Dynamics, Cambridge University Press, New York, 1998.

[6] R. REDHEFFER, Nonautonomous Lotka-Volterra system I, J. Differential Equations, 127 (1996), 519_ 540. 
Differential Equations \& Applications www.ele-math.com dea@ele-math.com 